Article

\title{
Towards a Real-Time Predictive Management Approach of Indoor Air Quality in Energy-Efficient Buildings
}

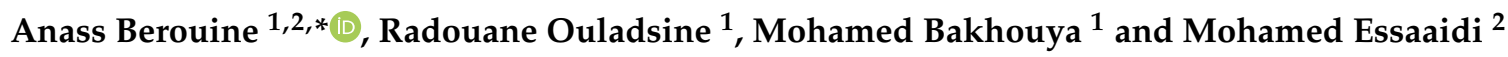 \\ 1 College of Engineering and Architecture, International University of Rabat, LERMA Lab, \\ Sala El Jadida 11100, Morocco; radouane.ouladsine@uir.ac.ma (R.O.); mohamed.bakhouya@uir.ac.ma (M.B.) \\ 2 ENSIAS, Mohamed V University, Rabat 10713, Morocco; essaaidi@ieee.org \\ * Correspondence: anass.berouine@uir.ac.ma; Tel.: +212-673-89-26-19
}

Received: 2 May 2020; Accepted: 2 June 2020; Published: 23 June 2020

check for updates

\begin{abstract}
Ventilation, heating and air conditioning systems are the main energy consumers in building sector. Improving the energy consumption of these systems, while satisfying the occupants' comfort, is the major concern of control and automation designers and researchers. Model predictive control (MPC) methods have been widely studied in order to reduce the energy usage while enhancing the occupants' comfort. In this paper, a generalized predictive control (GPC) algorithm based on controlled auto-regressive integrated moving average is investigated for standalone ventilation systems' control. A building's ventilation system is first modeled together with the GPC and MPC controllers. Simulations have been conducted for validation purposes and are structured into two main parts. In the first part, we compare the MPC with two traditional controllers, while the second part is dedicated to the comparison of the MPC against the GPC controller. Simulation results show the effectiveness of the GPC in reducing the energy consumption by about $4.34 \%$ while providing significant indoor air quality improvement.
\end{abstract}

Keywords: energy efficiency in buildings; indoor air quality comfort; $\mathrm{CO}_{2}$ regulation; ventilation systems control; model and generalized predictive control

\section{Introduction}

Heating, ventilation and air-conditioning (HVAC) systems represent approximately $50 \%$ of the global energy consumption in buildings and $36 \%$ of all energy-related $\mathrm{CO} 2$ emissions worldwide $[1,2]$. Therefore, building's systems, especially HVAC, have to be efficiently controlled in order to balance the tradeoff between the occupants' comfort and energy efficiency in buildings [3-5]. Four main metrics need to be considered, however, which are (i) the thermal comfort, (ii) visual comfort, (iii) acoustics comfort and (iv) the indoor air quality. According to a recent standard, integrating those metric parameters into the design of HVAC systems could provide a significant improvement of energy usage of these systems while ensuring comfortable indoor environmental conditions [6]. In brief, comfort metrics are required to assess the energy efficiency in buildings. In particular, indoor air quality which has been recognized as one of the most important factors influencing the indoor environmental quality of the occupants as well as one of the main sources of energy consumption in buildings [4,6], which depends mainly on the ventilation management service. On the other hand, ventilation systems in buildings are necessary to maintain a proper and healthy indoor air quality (IAQ) aiming to control the indoor environmental requirements, such as $\mathrm{CO}_{2}$ level, humidity and air velocity, by providing the required level of fresh air from outside to inside the building. The main aim is to reduce the excess of $\mathrm{CO}_{2}$ concentration with the respect of humidity by using an efficient ventilation rate in order to enhance 
the energy conservation while creating a more comfortable healthy environment. Therefore, developing advanced control strategies requires taking into consideration these environmental requirements, which are mostly based on international standards. For instance, Ashrae standard 62 is one of the important references that defines the performance requirements for ventilation to provide acceptable IAQ in buildings [3].

Indoor $\mathrm{CO}_{2}$ is considered among the most important parameters for developing intelligent control approaches of ventilation systems in order to minimize the overall energy usage while ensuring good indoor air quality. Basically, conventional building's ventilation systems have constant or prefixed ventilation rates based on the demand of that building's space. Typically, the fan acts automatically on the behalf of the occupants even when the demand for ventilation decreases. This could affect the thermal and indoor air quality comfort due to uncontrollable ventilation rate operations, leading to energy wastage. In addition, IAQ is considered among the main parameters for occupants' well-being and occupants' comfort in schools, libraries and other building sectors. In fact, inadequate ventilation systems, which do not supply enough fresh air, can cause poor IAQ leading to discomfort, symptoms and health issues. This might also affect the occupants' productivity [7]. In fact, our recent experimental study has shown a strong correlation between the $\mathrm{CO}_{2}$ concentration and the SPO2, the $\mathrm{O}_{2}$ level inside the occupants' blood [8]. The study showed also how the increase in $\mathrm{CO}_{2}$ concentration can affect the heart rate and SPO2 of the occupants leading to discomfort.

IAQ depends on many building context-awareness parameters, such as the envelope, operation sittings of the ventilation systems and the occupants' number [9]. Actually, most proposed methods for controlling IAQ is through the use of standalone ventilation systems by supplying the required outdoor air level using appropriate ventilation rates. These approaches will allow maintaining good indoor air quality while reducing the energy consumption [10-13]. Most of the deployed approaches are based on a fixed ventilation rate in order to minimize the power consumption while keeping a comfortable indoor air quality [14,15]. However, these approaches could operate efficiently in a static environment with priory known occupancy schedules. Recently, sophisticated control approaches have been proposed for environments with dynamic occupancy changes [16-18]. Namely, the well known proportional, integral and derivative (PID) control, which seems sufficient to cover the dynamic control requirements of these dynamic systems, however, the energy consumption remains an important issue [19]. Therefore, most recent studies focused on the parameter design of PID controllers in order to improve their performances, mainly in terms of precision, robustness and stability [20,21]. In fact, inappropriate setting of $\mathrm{P}, \mathrm{I}$ and $\mathrm{D}$ could affect the precision as well as the internal stability and robustness of the system [22]. Moreover, the PID controller is unsuitable for managing systems with multiple inputs/outputs with time-varying disturbances, such as in HVAC systems.

Advanced algorithms were recently developed in recent years for HVAC control systems, such as the fuzzy logic control, genetic algorithm and model predictive control (MPC) [23,24]. Among these control algorithms, predictive control strategies have been introduced as one of the most advanced control techniques used in building system control in order to regulate very complex related processes, such as in HVAC systems [25,26]-especially for energy and cost savings [27,28], robustness to disturbances and changes in operating conditions [29,30], indoor air quality and thermal comfort improvement $[31,32]$. In fact, model-based predictive control has an optimization mechanism that allows integrating the process's constraints and disturbances in its control action. The aim is to predict the dynamic behavior of the system, within a prediction horizon. Moreover, the MPC mechanism could handle uncertainties and nonlinear processes and their dynamics [33].

In our previous work, we have investigated the PID and ON/OFF controllers and validated their performance $[15,18]$. We then proposed a $\mathrm{CO}_{2}$-based strategy using a state feedback for controlling ventilation systems, and the results showed that the proposed state-feedback technique outperformed the Proportional Integral (PI) and ON/OFF controllers in terms of energy consumption while maintaining good indoor air quality. The three controllers have been deployed in our University testbed (EEBLab, for Energy Efficient Buildings Laboratory), which is located in Rabat, Morocco. The experiments were 
conducted in real sitting scenarios and the results showed that the $\mathrm{CO}_{2}$-based state feedback (SF) control was able to maintain the $\mathrm{CO}_{2}$ concentration in the comfortable zone, while minimizing energy consumption. It is worth noting that the EEBLab consists mainly of: (i) RES-Renewable Energy Sources (e.g., wind and solar), (ii) battery devices to store the excessive power, and (iii) active/passive systems (e.g., air conditioner, ventilation systems, geothermal, pad cooling system).

The work presented in this paper was conducted under two ongoing R\&D projects [34]. The aim was to develop predictive approaches and techniques for active/passive system (e.g., ventilation, HVAC, pad cooling, geothermal) control, including the integration of renewable energy and storage systems [35-37]. A platform that combined IoT and Big data processing technologies for real-time control of the building's systems was also deployed in our EEBLab for real testing [38]. In fact, in order to enable predictive control, a holistic platform, which integrates recent IoT sensing/actuating and Big data technologies, was developed for data monitoring and processing together with real-time machine-learning techniques. Mainly, the data streams we were collecting from a variety of sensors, such as $\mathrm{CO}_{2}$, temperature, humidity and motion sensors, will be used for predicting occupancy. Occupancy information will be then used to develop context-driven services and predictive control, such as controlling window opening and shading, smart HVAC, ventilation, and lighting services. Mainly, the aim was to carry out the MAPCAST closed loop (measure, analyze and predict the inputs control parameters for forecasting required actions), which enables the predictive control of active and passive systems including the control of our micro-grid systems [36,38].

The aim of the work presented in this paper was towards developing and designing advanced controllers of standalone ventilation systems by carrying out the MAPCAST principles. In fact, a real-time predictive management approach was then developed, which aimed to autonomously measure, analyze actual data, predict future values and forecast ventilation actions. The first part of this work was dedicated to ventilation's system modeling including the design of both generalized predictive control (GPC) and MPC controllers. Simulation results are then presented to compare first the MPC against the PI and SF control we have developed and compared in our previous work [39]. Mainly, in this work, a performance comparison between two predictive controllers, MPC and GPC, were performed and analyzed for a building's ventilation system. The PI and SF controllers were only used as baseline references to validate and assess the performance of MPC and GPC. The obtained results were analyzed and compared based on three main metrics, the regulation of indoor $\mathrm{CO}_{2}$ concentration, the ventilation rate, and the power consumption, both aggregated and disaggregated.

The outline of this article is organized as follows. Section 2 presents the proposed methodology followed throughout the article, which mainly describes the ventilation's control model using the MPC and GPC. Simulation parameters and evaluation metrics together with the obtained results are reported in Section 3. Section 4 provides the conclusions and perspectives.

\section{System Model and Methods}

This section introduces the model of the ventilation system for the air indoor regulation. The design and the models of the MPC and GPC controllers are then presented and discussed.

\subsection{Ventilation System Modeling}

The ventilation system was constituted of two standalone controlled fans, which were respectively responsible for supplying the fresh outdoor air into the inside of the building and draining the $\mathrm{CO}_{2}$ out of the building. In other words, these two fans were operating instantaneously under the same control actions in order to keep a good indoor air quality inside the building. The model describing this system was inspired from the $\mathrm{CO}_{2}$ mass balance method, which combines the indoor/outdoor $\mathrm{CO}_{2}$ concentration, the controlled air flow rate and the occupancy behavior [40]. This model was given by the following equation, where $\mathrm{Q}$ is the ventilation rate operation in $\left(\mathrm{m}^{3} / \mathrm{s}\right), \mathrm{N}$ is the number of occupants and $\mathrm{t}$ is the time in $(\mathrm{s}), \mathrm{V}$ represents the space's volume $\left(\mathrm{m}^{3}\right)$, q means the rate of the generated $\mathrm{CO}_{2}$ concentration per person $(\mathrm{L} / \mathrm{s})$, while $\mathrm{C}_{\text {ext }}\left(\right.$ resp. $\mathrm{C}_{\mathrm{i}}$ ) represents the external $\mathrm{CO}_{2}$ concentration 
(resp. internal), measured in ppm. The recommended internal $\mathrm{CO}_{2}$ concentration must be lower than 1000 ppm [41]:

$$
\mathrm{V} \frac{\mathrm{dC}_{\mathrm{i}}(\mathrm{t})}{\mathrm{dt}}=\mathrm{Q}(\mathrm{t})\left(\mathrm{C}_{\mathrm{i}}(\mathrm{t})-\mathrm{C}_{\mathrm{ext}}(\mathrm{t})\right)+\mathrm{qN}(\mathrm{t})
$$

This equation has a nonlinear behavior due to the inter-relationship between the $\mathrm{CO}_{2}$ concentration and the ventilations rates. It could be linearized as follows, where $\mathrm{Q}_{0}$ is a constant initial rate as well as the minimum ventilation rate, $\Delta \mathrm{Q}(\mathrm{t})$ is the change necessary to achieve the desired indoor $\mathrm{CO}_{2}$ level, $\mathrm{C}_{0}$ represents the initial indoor concentration of $\mathrm{C}_{\mathrm{i}}(\mathrm{t})$, and $\Delta \mathrm{C}_{\mathrm{i}}(\mathrm{t})$ is the concentration increment by applying $\Delta \mathrm{Q}(\mathrm{t})$ :

$$
\mathrm{Q}(\mathrm{t})=\mathrm{Q}_{0}+\Delta \mathrm{Q}(\mathrm{t}) \mathrm{C}_{\mathrm{i}}(\mathrm{t})=\mathrm{C}_{0}+\Delta \mathrm{C}_{\mathrm{i}}(\mathrm{t})
$$

Equation (1) can therefore be described by Equation (2):

$$
\dot{x}(t)=\frac{Q_{0}}{V} \Delta C_{i}(t)+\frac{\left(C_{0}-C_{e x t}\right)}{V} \Delta Q(t)+\frac{q N(t)}{V}
$$

Let us consider:

$$
\mathrm{A}_{\mathrm{c}}=\frac{\mathrm{Q}_{0}}{\mathrm{~V}} ; \mathrm{B}_{\mathrm{c}}=\frac{\left(\mathrm{C}_{0}-\mathrm{C}_{\mathrm{ext}}\right)}{\mathrm{V}} ; \mathrm{x}=\Delta \mathrm{C}_{\mathrm{i}}(\mathrm{t}) ; \mathrm{u}(\mathrm{t})=\Delta \mathrm{Q}(\mathrm{t}) ;
$$

where $\mathrm{qN}(\mathrm{t})=\mathrm{F}(\mathrm{t})$ is supposed as human disturbance, which must be integrated as an input parameter to the proposed system. Finally, the state-space model is the following:

$$
\dot{x}(t)=A_{c} x(t)+B_{c} u(t)+\frac{F(t)}{V}
$$

\subsection{Predictive Controllers Design}

In this sub-section, we introduce the MPC and GPC models. The aim is to show how these controllers could maintain the system model based on input/output ventilation rates and carbon dioxide concentrations in the building (Equation (3)).

\subsubsection{MPC Controller Design}

In order to design the MPC, a discrete model form of our system (Equation (3)) is required. In fact, the obtained Euler approximation has the following discrete state space equation:

$$
\left\{\begin{array}{c}
x(k+1)=A x(k)+B u(k) \\
y(k+1)=C x(k+1)
\end{array}\right.
$$

The representation of n-step-ahead of Equation (4) is given by the following matrix-based forms (i.e., the system's state, output, and input control increment):

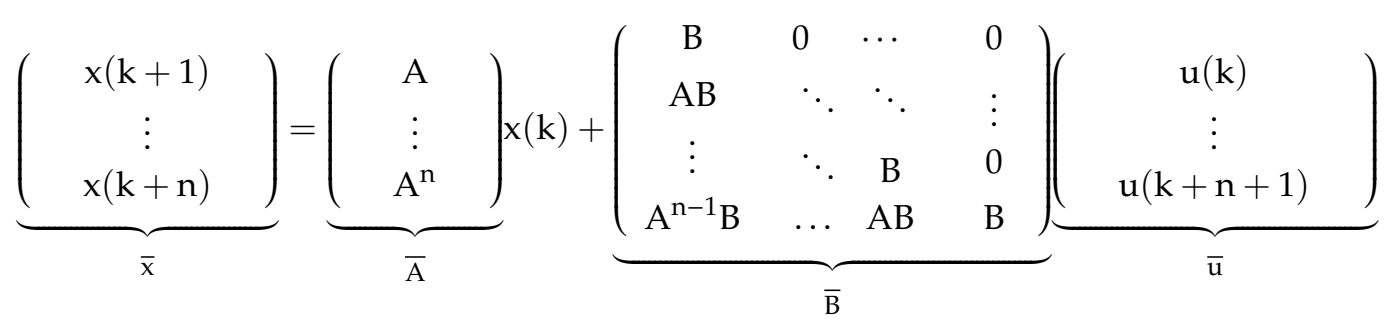




$$
\begin{aligned}
& \underbrace{\left(\begin{array}{c}
\mathrm{y}(\mathrm{k}+1) \\
\vdots \\
\mathrm{y}(\mathrm{k}+\mathrm{n})
\end{array}\right)}_{\overline{\mathrm{y}}}=\underbrace{\left(\begin{array}{c}
\mathrm{CA} \\
\vdots \\
\mathrm{CA}^{\mathrm{n}}
\end{array}\right)}_{\overline{\mathrm{C}}} \times \mathrm{k})+\underbrace{\left(\begin{array}{cccc}
\mathrm{CB} & 0 & \cdots & 0 \\
\mathrm{CAB} & \ddots & \ddots & \vdots \\
\vdots & \ddots & \mathrm{CB} & 0 \\
\mathrm{CA}^{\mathrm{n}-1} \mathrm{~B} & \ldots & \mathrm{CAB} & \mathrm{CB}
\end{array}\right)}_{\overline{\mathrm{D}}} \underbrace{\left(\begin{array}{c}
\mathrm{u}(\mathrm{k}+1) \\
\vdots \\
\mathrm{u}(\mathrm{k}+\mathrm{n}+1)
\end{array}\right)}_{\overline{\mathrm{u}}} \\
& \underbrace{\left(\begin{array}{c}
\mathrm{u}(\mathrm{k}) \\
\vdots \\
\mathrm{u}(\mathrm{k}+\mathrm{n})
\end{array}\right)}_{\overline{\mathrm{u}}}=\underbrace{\left(\begin{array}{c}
1 \\
\vdots \\
1^{\mathrm{n}}
\end{array}\right)}_{\mathrm{I}_{1}} \mathrm{u}(\mathrm{k}-1)+\underbrace{\left(\begin{array}{cccc}
1 & 0 & \cdots & 0 \\
1 & \ddots & \ddots & \vdots \\
\vdots & \ddots & \ddots & 0 \\
& \ldots & 1 & 1
\end{array}\right)}_{\mathrm{I}_{2}} \underbrace{\left(\begin{array}{c}
\Delta \mathrm{u}(\mathrm{k}) \\
\Delta \mathrm{u}(\mathrm{k}+1) \\
\mathrm{u}(\mathrm{k}+\mathrm{n}+1)
\end{array}\right)}_{\Delta \overline{\mathrm{u}}(\mathrm{k})}
\end{aligned}
$$

Replacing Equation (7) in (5), we obtain the predictive model: $\bar{x}=\overline{\mathrm{A}} \mathrm{x}(\mathrm{k})+\overline{\mathrm{BI}}_{1} \mathrm{u}(\mathrm{k}-1)+\overline{\mathrm{B}} \mathrm{I}_{2} \Delta \overline{\mathrm{u}}(\mathrm{k})$. $\overline{\mathrm{DI}}_{2} \Delta \overline{\mathrm{u}}(\mathrm{k})$.

Replacing Equation (7) in (6), we get the predictive observation model: $\bar{y}=\overline{\mathrm{C}} \mathrm{x}(\mathrm{k})+\overline{\mathrm{DI}}_{1} \mathrm{u}(\mathrm{k}-1)+$

The control objective is to find the future incremental action $\Delta \overline{\mathrm{u}}(\mathrm{k})$, which is able to reach the desired predicted reference trajectory presented by the following equation:

$$
\mathrm{y}_{\text {ref }}=\left[\mathrm{y}_{\text {ref }}(\mathrm{k}+1), \mathrm{y}_{\text {ref }}(\mathrm{k}+2), \ldots, \mathrm{y}_{\text {ref }}(\mathrm{k}+\mathrm{n})\right]
$$

In fact, the error between the set point references and the predicted system's outputs is: $E=y-y_{\text {ref: }}$. Thus, the predicted control action $\Delta \overline{\mathrm{u}}$ that minimizes this error is computed by minimizing the following criterion:

$$
\mathrm{J}=\frac{1}{2}\left(\mathrm{EQE}^{\mathrm{T}}+\Delta \overline{\mathrm{u}} \mathrm{R} \Delta \overline{\mathrm{u}}^{\mathrm{T}}\right)
$$

\subsubsection{GPC Controller Design}

The basic theory of the GPC method, as described in [42], is to compute a sequence of future control actions in order to minimize a multistage cost function defined over a prediction horizon. The criterion index to be optimized is the expectation of a quadratic function, measuring the distance between the predicted system's outputs and some predicted reference sequence over a prediction horizon and a quadratic function measuring the control effort. Due to its correctness and effectiveness, the GPC has become the most popular MPC method used in various applications both in academia and industrial processes $[43,44]$.

The most transferred function model used by the GPC algorithm is called CARIMA (controlled auto-regressive integrated moving average) model. This form of plant model is that the uncertainty is added into a good representation, so that the slow variation of disturbances could have a non-zero steady-state. Moreover, this model is compact and easy to handle:

$$
\mathrm{A}\left(\mathrm{z}^{-1}\right) \mathrm{y}(\mathrm{k})=\mathrm{B}\left(\mathrm{z}^{-1}\right) \mathrm{u}(\mathrm{k})+\frac{\mathrm{T}\left(\mathrm{z}^{-1}\right)}{\Delta} \mathrm{d}(\mathrm{k})
$$

where $\mathrm{u}$ and $\mathrm{y}$ are the control inputs and the system output sequences of the plant, respectively, $\mathrm{A}$, $\mathrm{B}$ and $\mathrm{T}$ are the polynomial Z-function, $\Delta$ is the variation operator $\Delta \mathrm{u}_{\mathrm{k}}=\mathrm{u}(\mathrm{k})-\mathrm{u}(\mathrm{k}-1)$, and $\mathrm{d}(\mathrm{k})$ represents the disturbance (white noise). Therefore, the main objective is to convert our state-space model into CARIMA form as follows:

$$
\left\{\begin{array}{c}
x(k+1)=A x(k)+B u(k)+d(k) \\
y(k+1)=C x(k+1)
\end{array}\right.
$$


By applying the Z-transform representations, we obtain:

$$
\left\{\begin{array}{c}
x\left(z^{-1}\right)=A z^{-1} x\left(z^{-1}\right)+B z^{-1} u\left(z^{-1}\right)+z^{-1} d\left(z^{-1}\right) \\
y\left(z^{-1}\right)=C x\left(z^{-1}\right)
\end{array}=>\left\{\begin{array}{c}
x\left(z^{-1}\right)=\frac{B z^{-1}}{1-A z^{-1}} u\left(z^{-1}\right)+\frac{z^{-1}}{1-A z^{-1}} d\left(z^{-1}\right) \\
y\left(z^{-1}\right)=C x\left(z^{-1}\right)
\end{array}\right.\right.
$$

Therefore:

$$
y\left(z^{-1}\right)=\frac{C B z^{-1}}{1-A z^{-1}} u\left(z^{-1}\right)+\frac{C z^{-1}}{1-A z^{-1}} d\left(z^{-1}\right)
$$

Hence:

$$
1-\mathrm{Az}^{-1} \mathrm{y}\left(\mathrm{z}^{-1}\right)=\mathrm{CBz}^{-1} \mathrm{u}\left(\mathrm{z}^{-1}\right)+\frac{\mathrm{Cz}^{-1}\left(1-\mathrm{z}^{-1}\right)}{1-\mathrm{z}^{-1}} \mathrm{~d}\left(\mathrm{z}^{-1}\right)
$$

where:

$$
\left\{\begin{array}{c}
\mathrm{A}\left(\mathrm{z}^{-1}\right)=1-\mathrm{Az}^{-1} \\
\mathrm{~B}\left(\mathrm{z}^{-1}\right)=\mathrm{CBz}^{-1} \\
\mathrm{~T}\left(\mathrm{z}^{-1}\right)=\mathrm{Cz}^{-1}\left(1-\mathrm{z}^{-1}\right) \\
\Delta=1-\mathrm{z}^{-1}
\end{array}\right.
$$

Thus, the CARIMA form can be described as follows:

$$
\mathrm{A}\left(\mathrm{z}^{-1}\right) \mathrm{y}\left(\mathrm{z}^{-1}\right)=\mathrm{B}\left(\mathrm{z}^{-1}\right) \mathrm{u}\left(\mathrm{z}^{-1}\right)+\frac{\mathrm{T}\left(\mathrm{z}^{-1}\right)}{\Delta} \mathrm{d}\left(\mathrm{z}^{-1}\right) .
$$

We then synthetize from the CARIMA model a one-step-ahead prediction equation, which represents a set of simultaneous prediction equations:

$$
\begin{gathered}
\mathrm{A}\left(\mathrm{z}^{-1}\right) \Delta \mathrm{y}\left(\mathrm{z}^{-1}\right)=\mathrm{B}\left(\mathrm{z}^{-1}\right) \Delta \mathrm{u}\left(\mathrm{z}^{-1}\right)+\mathrm{T}\left(\mathrm{z}^{-1}\right) \mathrm{d}\left(\mathrm{z}^{-1}\right), \\
\mathrm{A}\left(\mathrm{z}^{-1}\right)=\left(1-\mathrm{A}\left(\mathrm{z}^{-1}\right)\right)\left(1-\mathrm{z}^{-1}\right)=1+\mathrm{A}_{1}\left(\mathrm{z}^{-1}\right)+\mathrm{A}_{2}\left(\mathrm{z}^{-1}\right),
\end{gathered}
$$

Therefore, our model design is:

$$
\mathrm{A}\left(\mathrm{z}^{-1}\right) \mathrm{y}\left(\mathrm{z}^{-1}\right)=\mathrm{b}\left(\mathrm{z}^{-1}\right) \Delta \mathrm{u}\left(\mathrm{z}^{-1}\right) .
$$

The simultaneous prediction equations after n-steps-ahead can be considered as follows:

$$
\left\{\begin{array}{l}
\mathrm{k}+1: \mathrm{y}(\mathrm{k}+1)+\mathrm{A}_{1} \mathrm{y}(\mathrm{k})+\mathrm{A}_{2} \mathrm{y}(\mathrm{k}-1)=\mathrm{CB} \Delta \mathrm{u}(\mathrm{k}) \\
\mathrm{k}+2: \mathrm{y}(\mathrm{k}+2)+\mathrm{A}_{1} \mathrm{y}(\mathrm{k}+1)+\mathrm{A}_{2} \mathrm{y}(\mathrm{k})=\mathrm{CB} \Delta \mathrm{u}(\mathrm{k}+1) \\
\mathrm{k}+3: \mathrm{y}(\mathrm{k}+3)+\mathrm{A}_{1} \mathrm{y}(\mathrm{k}+2)+\mathrm{A}_{2} \mathrm{y}(\mathrm{k}+1)=\mathrm{CB} \Delta \mathrm{u}(\mathrm{k}+2) \\
\quad \vdots \\
\mathrm{k}+\mathrm{n}: \mathrm{y}(\mathrm{k}+\mathrm{n})+\mathrm{A}_{1} \mathrm{y}(\mathrm{k}+\mathrm{n}-1)+\mathrm{A}_{2} \mathrm{y}(\mathrm{k}+\mathrm{n}-2)=\mathrm{CB} \Delta \mathrm{u}(\mathrm{k}+\mathrm{n}-1)
\end{array}\right.
$$

The idea is to arrange the above equations into a prediction matrix form, which is simple and easy to handle as follows:

$$
\underbrace{\mathrm{C}_{\mathrm{A}}\left[\begin{array}{c}
\mathrm{y}(\mathrm{k}+1) \\
\mathrm{y}(\mathrm{k}+2) \\
\mathrm{y}(\mathrm{k}+3) \\
\vdots \\
\mathrm{y}(\mathrm{k}+\mathrm{n})
\end{array}\right]}_{\mathrm{y}_{(\mathrm{k})}^{\mathrm{F}}}+\underbrace{\mathrm{H}_{\mathrm{A}}\left[\begin{array}{c}
\mathrm{y}(\mathrm{k}) \\
\mathrm{y}(\mathrm{k}+1) \\
\mathrm{y}(\mathrm{k}+2) \\
\vdots \\
\mathrm{y}(\mathrm{k}+\mathrm{n}-1)
\end{array}\right]}_{\mathrm{y}_{(\mathrm{k})}^{\mathrm{P}}}=\underbrace{\mathrm{C}_{\mathrm{B}}\left[\begin{array}{c}
\Delta \mathrm{u}(\mathrm{k}) \\
\Delta \mathrm{u}(\mathrm{k}+1) \\
\Delta \mathrm{u}(\mathrm{k}+2) \\
\vdots \\
\Delta \mathrm{u}(\mathrm{k}+\mathrm{n}-1)
\end{array}\right]}_{\Delta \mathrm{u}_{(\mathrm{k})}^{\mathrm{F}}}+\underbrace{\mathrm{H}_{\mathrm{B}}\left[\begin{array}{c}
\Delta \mathrm{u}(\mathrm{k}-1) \\
\Delta \mathrm{u}(\mathrm{k}-2) \\
\Delta \mathrm{u}(\mathrm{k}-3) \\
\vdots \\
\Delta \mathrm{u}(\mathrm{k}-\mathrm{n})
\end{array}\right]}_{\Delta \mathrm{u}_{(\mathrm{k})}^{\mathrm{P}}}
$$


where $\mathrm{y}_{(\mathrm{k})}^{\mathrm{F}}, \mathrm{y}_{(\mathrm{k})}^{\mathrm{P}}, \Delta \mathrm{u}_{(\mathrm{k})}^{\mathrm{F}}$ and $\Delta \mathrm{u}_{(\mathrm{k})}^{\mathrm{P}}$ are the unknown prediction output, known past output, future input (decision variable) to be calculated, and the known past input, respectively. $\mathrm{C}_{\mathrm{A}}, \mathrm{H}_{\mathrm{A}}, \mathrm{C}_{\mathrm{B}} \mathrm{H}_{\mathrm{B}}$ are the key matrices of our predictive control:

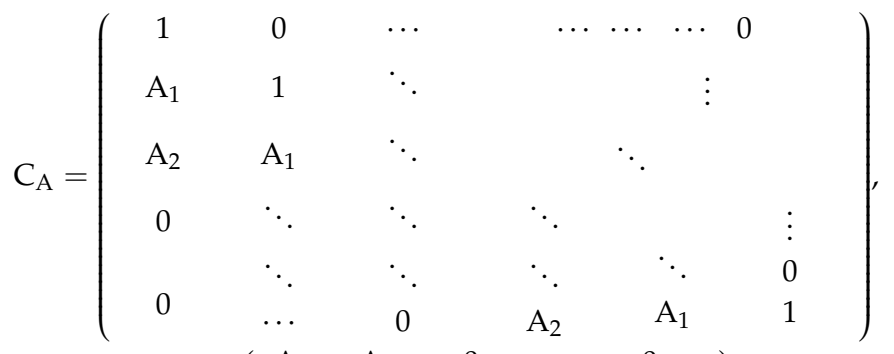

$$
\begin{aligned}
& \mathrm{H}_{\mathrm{A}}=\left(\begin{array}{ccccc}
\mathrm{A}_{1} & \mathrm{~A}_{2} & 0 & \cdots & 0 \\
\mathrm{~A}_{2} & 0 & 0 & \cdots & 0 \\
0 & 0 & \ddots & \ddots & \vdots \\
\vdots & \ddots & \ddots & \ddots & 0 \\
0 & \cdots & \cdots & \cdots & 0
\end{array}\right) \\
& \mathrm{C}_{\mathrm{B}}=\left(\begin{array}{cccc}
\mathrm{CB} & 0 & \cdots & 0 \\
0 & \mathrm{CB} & \ddots & \vdots \\
\vdots & \ddots & \ddots & 0 \\
0 & \cdots & 0 & \mathrm{CB}
\end{array}\right), \\
& \mathrm{H}_{\mathrm{B}}=\left(\begin{array}{ccc}
0 & \cdots & 0 \\
\vdots & \ddots & \vdots \\
0 & \cdots & 0
\end{array}\right) \text {. }
\end{aligned}
$$

As follows, the output predictions using the shorthand notation for more compact representation:

$$
\mathrm{C}_{\mathrm{A}} \mathrm{y}_{(\mathrm{k})}^{\mathrm{F}}+\mathrm{H}_{\mathrm{A}} \mathrm{y}_{(\mathrm{k})}^{\mathrm{P}}=\mathrm{C}_{\mathrm{B}} \Delta \mathrm{u}_{(\mathrm{k})}^{\mathrm{F}}+\mathrm{H}_{\mathrm{B}} \Delta \mathrm{u}_{(\mathrm{k})}^{\mathrm{P}}, \mathrm{y}_{(\mathrm{k})}^{\mathrm{F}}=\underbrace{\mathrm{C}_{\mathrm{A}}^{-1} \mathrm{C}_{\mathrm{B}}}_{\mathrm{H}} \Delta \mathrm{u}_{(\mathrm{k})}^{\mathrm{F}}+\underbrace{\mathrm{C}_{\mathrm{A}}^{-1} \mathrm{H}_{\mathrm{B}}}_{\mathrm{P}} \Delta \mathbf{u}_{(\mathrm{k})}^{\mathrm{P}}-\underbrace{\mathrm{C}_{\mathrm{A}}^{-1} \mathrm{H}_{\mathrm{A}} \mathrm{y}_{(\mathrm{k})}^{\mathrm{P}}}_{\mathrm{Q}}
$$

Let us now define the criterion to be optimized, which mainly relies in our case on error change, between the predicted system output $\mathrm{y}_{(\mathrm{k})}^{\mathrm{F}}$ and the predicted reference sequence $\mathrm{r}_{(\mathrm{k})}^{\mathrm{F}}=$ $\left[\mathrm{r}_{\mathrm{k}+1}, \mathrm{r}_{\mathrm{k}+2}, \mathrm{r}_{\mathrm{k}+3}, \cdots \mathrm{r}_{\mathrm{k}+\mathrm{n}}\right]$ over the horizon, hence the error is $\mathrm{e}_{(\mathrm{k})}^{\mathrm{F}}=\mathrm{r}_{(\mathrm{k})}^{\mathrm{F}}-\mathrm{y}_{(\mathrm{k})}^{\mathrm{F}}$, and consequently the optimal cost criterion is the following:

$$
\mathrm{J}=\left(\mathrm{e}_{(\mathrm{k})}^{\mathrm{F}}\right)^{\mathrm{T}} \cdot \mathrm{e}_{(\mathrm{k})}^{\mathrm{F}}+\lambda\left(\Delta \mathrm{u}_{(\mathrm{k})}^{\mathrm{F}}\right)^{\mathrm{T}} \Delta \mathrm{u}_{(\mathrm{k})}^{\mathrm{F}}
$$

where $\lambda$ is the control weighting factor that reduces the magnitude of the control increments in the cost function. This criterion is optimized through the calculation of the gradient $\Delta \mathrm{u}_{(\mathrm{k})}^{\mathrm{F}}$ as follows:

By replacing the error change $\mathrm{e}_{(\mathrm{k})}^{\mathrm{F}}=\mathrm{r}_{(\mathrm{k})}^{\mathrm{F}}-\mathrm{y}_{(\mathrm{k})}^{\mathrm{F}}$ into (12), we obtain:

$$
\begin{gathered}
\mathrm{J}=\left[\mathrm{r}_{(\mathrm{k})}^{\mathrm{F}}-\mathrm{H} \Delta \mathrm{u}_{(\mathrm{k})}^{\mathrm{F}}-\mathrm{P} \Delta \mathrm{u}_{(\mathrm{k})}^{\mathrm{P}}-\mathrm{Q} \Delta \mathrm{y}_{(\mathrm{k})}^{\mathrm{P}}\right]^{\mathrm{T}} \times\left[\mathrm{r}_{(\mathrm{k})}^{\mathrm{F}}-\mathrm{H} \Delta \mathrm{u}_{(\mathrm{k})}^{\mathrm{F}}-\mathrm{P} \Delta \mathrm{u}_{(\mathrm{k})}^{\mathrm{P}}-\mathrm{Q} \Delta \mathrm{y}_{(\mathrm{k})}^{\mathrm{P}}\right]+\lambda\left(\Delta \mathrm{u}_{(\mathrm{k})}^{\mathrm{F}}\right)^{\mathrm{T}} \Delta \mathrm{u}_{(\mathrm{k})}^{\mathrm{F}} \\
\mathrm{J}=\left[\left(\mathrm{H} \Delta \mathrm{u}_{(\mathrm{k})}^{\mathrm{F}}\right)^{\mathrm{T}}\left(\mathrm{H} \Delta \mathrm{u}_{(\mathrm{k})}^{\mathrm{F}}\right)-\left(2\left(\mathrm{H} \Delta \mathrm{u}_{(\mathrm{k})}^{\mathrm{F}}\right)^{\mathrm{T}}\left(\mathrm{r}_{(\mathrm{k})}^{\mathrm{F}}-\mathrm{P} \Delta \mathrm{u}_{(\mathrm{k})}^{\mathrm{P}}-\mathrm{Q} \Delta \mathrm{y}_{(\mathrm{k})}^{\mathrm{P}}\right)\right)\right]+\lambda\left(\Delta \mathrm{u}_{(\mathrm{k})}^{\mathrm{F}}\right)^{\mathrm{T}} \Delta \mathrm{u}_{(\mathrm{k})}^{\mathrm{F}}
\end{gathered}
$$

Then, we applied the mathematical rules:

$$
\left\{\begin{array}{c}
\nabla_{X}\left(a^{T} X\right)=a \\
\nabla_{X}\left(X^{T} S X\right)=\left(S+S^{T}\right) X
\end{array} ; \nabla_{X}\left(X^{T} a\right)=a\right.
$$


We get:

$$
\begin{aligned}
& \nabla_{\Delta u_{(\mathrm{k})}^{\mathrm{F}}} \mathrm{J}=\nabla_{\Delta \mathrm{u}_{(\mathrm{k})}^{\mathrm{F}}}[\underbrace{\left(\Delta \mathrm{u}_{(\mathrm{k})}^{\mathrm{F}}\right)^{\mathrm{T}}}_{\mathrm{X}} \underbrace{\left(\mathrm{H}^{\mathrm{T}} \mathrm{H}+\lambda \mathrm{I}\right)}_{\mathrm{S}} \underbrace{\Delta \mathrm{u}_{(\mathrm{k})}^{\mathrm{F}}}_{\mathrm{X}}-2\left(\mathrm{H} \Delta \mathrm{u}_{(\mathrm{k})}^{\mathrm{F}}\right)^{\mathrm{T}} \mathrm{H}^{\mathrm{T}} \underbrace{\left(\mathrm{r}_{(\mathrm{k})}^{\mathrm{F}}-\mathrm{P} \Delta \mathrm{u}_{(\mathrm{k})}^{\mathrm{P}}-\mathrm{Q} \Delta \mathrm{y}_{(\mathrm{k})}^{\mathrm{P}}\right)}_{\mathrm{a}}] \\
& \nabla_{\Delta \mathrm{u}_{(\mathrm{k})}^{\mathrm{F}} \mathrm{J}}=\left(\mathrm{S}+\mathrm{S}^{\mathrm{T}}\right) \Delta \mathrm{u}_{(\mathrm{k})}^{\mathrm{F}}-2 \mathrm{H}^{\mathrm{T}}\left(\mathrm{r}_{(\mathrm{k})}^{\mathrm{F}}-\mathrm{P} \Delta \mathrm{u}_{(\mathrm{k})}^{\mathrm{P}}-\mathrm{Q} \Delta \mathrm{y}_{(\mathrm{k})}^{\mathrm{P}}\right)=0 \\
& \Delta \mathrm{u}_{(\mathrm{k})}^{\mathrm{F}}=\mathrm{S}^{-1} \mathrm{H}^{\mathrm{T}}\left(\mathrm{r}_{(\mathrm{k})}^{\mathrm{F}}-\mathrm{P} \Delta \mathrm{u}_{(\mathrm{k})}^{\mathrm{P}}-\mathrm{Qy} \mathrm{y}_{(\mathrm{k})}^{\mathrm{P}}\right) .
\end{aligned}
$$

In order to implement this control for real-time simulations, we note that the relation must be adapted for the feedback design as follows:

$$
\begin{gathered}
\Delta \mathrm{u}_{(\mathrm{k})}^{\mathrm{F}}=\mathrm{Mr}_{(\mathrm{k})}^{\mathrm{F}}-\mathrm{N} \Delta \mathrm{u}_{(\mathrm{k})}^{\mathrm{P}}-\mathrm{Ly}_{(\mathrm{k})}^{\mathrm{P}} \\
\Delta \mathrm{u}_{(\mathrm{k})}^{\mathrm{F}}+\mathrm{N} \Delta \mathrm{u}_{(\mathrm{k})}^{\mathrm{P}}=\mathrm{Mr}_{(\mathrm{k})}^{\mathrm{F}}-\mathrm{Ly}_{(\mathrm{k})}^{\mathrm{P}} \\
\left\{\begin{array}{c}
\mathrm{Mr}_{(\mathrm{k})}^{\mathrm{F}}=\mathrm{M}_{1} \mathrm{zr}_{(\mathrm{k})}+\mathrm{M}_{2} \mathrm{z}^{2} \mathrm{r}_{(\mathrm{k})}+\mathrm{M}_{3} \mathrm{z}^{3} \mathrm{r}_{(\mathrm{k})}+\cdots \mathrm{M}_{\mathrm{n}} \mathrm{z}^{\mathrm{n}} \mathrm{r}_{(\mathrm{k})}+=\mathrm{M}(\mathrm{z}) \mathrm{r}_{(\mathrm{k})} \\
\mathrm{Ly}_{(\mathrm{k})}^{\mathrm{P}}=\mathrm{L}_{0} \mathrm{y}_{(\mathrm{k})}+\mathrm{L}_{1} \mathrm{z}^{-1} \mathrm{y}_{(\mathrm{k})}+\mathrm{L}_{2} \mathrm{z}^{-2} \mathrm{y}_{(\mathrm{k})}+\cdots+\mathrm{L}_{\mathrm{n}-1} \mathrm{z}^{-\mathrm{n}-1} \mathrm{y}_{(\mathrm{k})}=\mathrm{L}\left(\mathrm{z}^{-1}\right) \mathrm{y}_{(\mathrm{k})} \\
\Delta \mathrm{u}_{(\mathrm{k})}^{\mathrm{F}}+\mathrm{N} \Delta \mathrm{u}_{(\mathrm{k})}^{\mathrm{P}}=\mathrm{D}\left(\mathrm{z}^{-1}\right) \Delta \mathrm{u}_{(\mathrm{k})}
\end{array}\right.
\end{gathered}
$$

Hence, the schema bloc of the controlled system by the GPC-based CARIMA model is described in Figure 1:

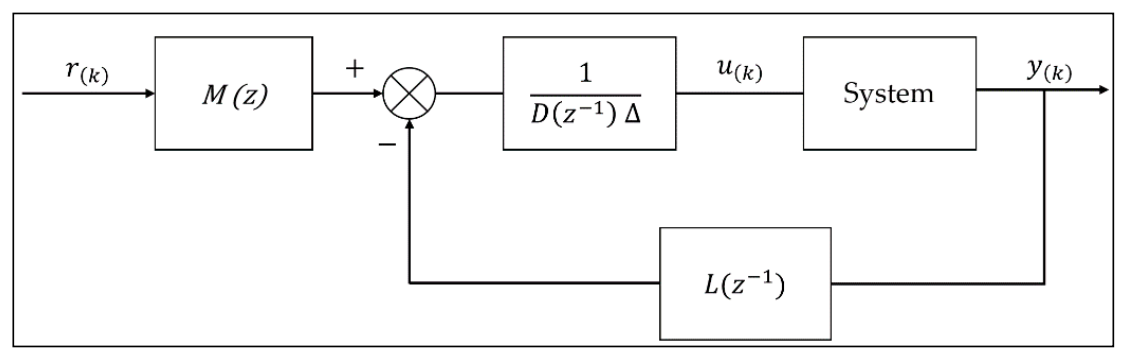

Figure 1. System's controller feedback blocs of the generalized predictive control (GPC)-based controlled auto-regressive integrated moving average (CARIMA) model.

\section{Results and Discussion}

This section introduces the implementation and performance evaluation of the MPC and GPC for the ventilation system's control. Matlab/Simulink was used as a platform for computing and implementing the MPC and GPC models. The controllers' parameters were obtained by computing different testing, taking into consideration the settling time, rise time and the overshoot of the $\mathrm{CO}_{2}$ concentration, which were chosen by the suitable tuning as illustrated in Table 1. Concerning the PI and SF controllers, the tuning process together with their performance evaluation were detailed in our previous work [39]. The gain values we used are $\mathrm{Kp}=-0.3$ and $\mathrm{Ti}=7.5$, for proportional gain and integral time, respectively. In addition, numerical simulations were conducted under the same conditions as presented in Table 2, which describes the parameters and the building's ventilation system, respectively. We used the features and characteristics of the ventilation system already deployed in our test site (EEBLab). Mainly, the ventilators were operating at a maximum air flow rate of about $0.6 \mathrm{~m}^{3 / \mathrm{s}}$ and were supplied by a solar photovoltaic system $(24 \mathrm{~V}, \mathrm{DC})$. The simulations were performed during a time period of about $15,000 \mathrm{~s}$ using same parameters and conditions. 
Table 1. Input tuning of the model predictive control (MPC) and GPC algorithms' parameters.

\begin{tabular}{ccccc}
\hline Input Parameters & $\begin{array}{c}\text { Control Horizon } \\
\left(N_{u}\right)\end{array}$ & $\begin{array}{c}\text { Prediction } \\
\left(N_{p}\right)\end{array}$ & $\begin{array}{c}\text { Sampling Time } \\
\left(T_{s}\right)\end{array}$ & $\begin{array}{c}\text { Weighting Control } \\
(\lambda)\end{array}$ \\
\hline Value & 3 & 15 & $10 \mathrm{~s}$ & 0.6 \\
\hline
\end{tabular}

Table 2. Settings of the ventilation system model parameters.

\begin{tabular}{cccc}
\hline Parameters Name & Parameter Description & Value & Unit \\
\hline$V$ & Simulated space volume & 400 & $\mathrm{~m}^{3}$ \\
$q$ & $\mathrm{CO}_{2}$ generation rate per person & 0.0086 & $1 / \mathrm{s}$ \\
$Q_{0}$ & Minimum ventilation rate & $1 / 3600$ & $\mathrm{~m}^{3} / \mathrm{s}$ \\
$Q_{\max }$ & Maximum ventilation rate & 0.6 & $\mathrm{~m}^{3} / \mathrm{s}$ \\
$C_{e x t}$ & Outdoor $\mathrm{CO}_{2}$ concentration & 450 & $\mathrm{ppm}$ \\
$C_{0}$ & Initial indoor $\mathrm{CO}_{2}$ concentration & 700 & $\mathrm{ppm}$ \\
$C_{s e t}$ & Setpoint of $\mathrm{CO}_{2}$ concentration & 750 & $\mathrm{ppm}$ \\
$N_{\max }$ & Maximum number of occupants & 100 & - \\
$\Delta_{p}$ & Fan inlet/outlet increment total pressure & 1500 & $\mathrm{~Pa}$ \\
$\mu$ & Overall efficiency & 0.65 & - \\
\hline
\end{tabular}

In order to assess the performance of the $\mathrm{CO}_{2}$-based GPC control strategy with the MPC, PI and SF control approaches, four main metrics were evaluated: (a) the indoor $\mathrm{CO}_{2}$ regulation, (b) the ventilation rate operation of the controlled system in $\left(\mathrm{m}^{3} / \mathrm{s}\right)$, (c) the instantaneous electricity power, and (d) the total electricity power consumed by the system in $(\mathrm{kW} / \mathrm{h})$, which was calculated using the following equation presented in [45]:

$$
\mathrm{PC}_{\text {elec }}=\mathrm{Q}_{\mathrm{i}} \times \Delta \mathrm{p} \times\left(\frac{\mathrm{t}}{3600}\right) \times\left(\frac{\mu}{1000}\right)
$$

where $\mathrm{PC}_{\text {elec }}$ is the power consumption in $(\mathrm{kW} / \mathrm{h}), \mathrm{Q}_{\mathrm{i}}$ is the ventilation rate in $\left(\mathrm{m}^{3} / \mathrm{s}\right), \Delta \mathrm{p}$ is the total pressure rise from the fan inlet to the outlet in $(\mathrm{Pa}), \mathrm{t}$ is the time in (s) and $\mu$ is the overall efficiency of the fan.

An indoor $\mathrm{CO}_{2}$ concentration scenario generated by the occupants was used as the human disturbance as well as a forecast input for the MPC and GPC. As illustrated in Figure 2, the generated $\mathrm{CO}_{2}$ concentration (ppm) was estimated according to the occupants' number using the transfer function presented in [46]. The occupied building for our case study was considered a university lecture room with a maximum occupation of 100 students. The estimated occupancy count of the lecture room was drawn from our real-life knowledge of the building usage during the time period 15,000 s, which was composed of three periods: a $1 \mathrm{~h} 40 \mathrm{~min}$ lecture, $1 \mathrm{~h} 10 \mathrm{~min}$ break, and then another $1 \mathrm{~h} 40 \mathrm{~min}$ lecture.

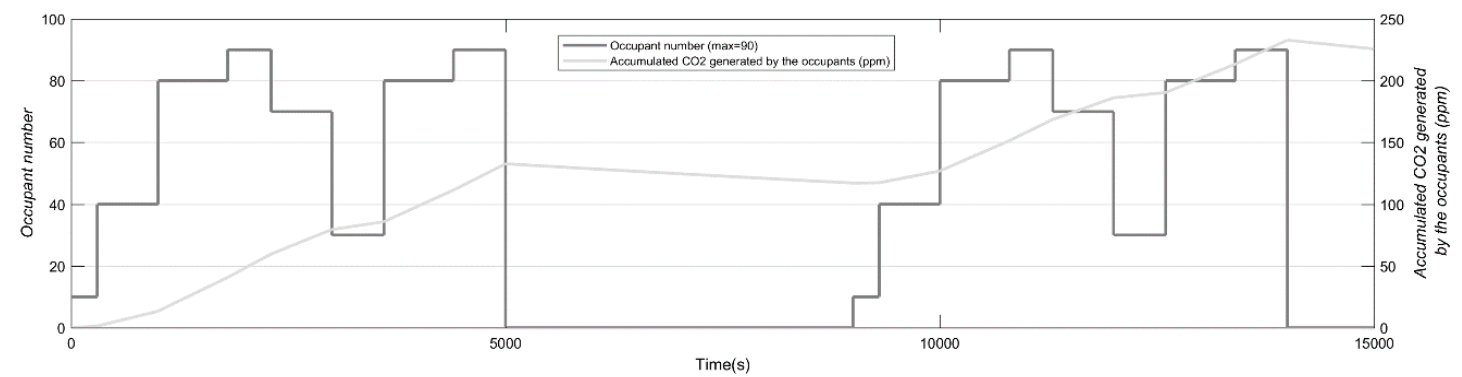

Figure 2. Generated indoor $\mathrm{CO} 2$ concentration used as the forecast input for the MPC and GPC.

The rest of this sub-section presents the obtained results using the aforementioned simulation settings. First, the MPC controller is evaluated and compared against the PI and SF, while the second focuses on the comparison between the two predictive controllers, the MPC and GPC. 


\subsection{MPC vs. PI and SF}

Figure 3 depicts the comparison between the MPC against the PI and SF controllers. Figure 3a shows the regulation of the $\mathrm{CO}_{2}$ concentration together with the occupancy number, the accumulated $\mathrm{CO}_{2}$ concentration generated by the occupants and the $\mathrm{CO}_{2}$ concentration reference (750 ppm). As shown in this figure, the regulation of the indoor $\mathrm{CO}_{2}$ concentration based on the MPC method strategy allowed the higher performance compared to the PI and SF control techniques. In fact, the MPC controller performed well, as expected, as it was able to reach and follow the $\mathrm{CO}_{2}$ reference and to maintain the comfort level despite the disturbance introduced by the occupants. On the other hand, the PI and SF showed an acceptable overshoot since the constraint range on the indoor $\mathrm{CO}_{2}$ concentration were fixed to $\pm 10 \mathrm{ppm}$ around the $\mathrm{CO}_{2}$ reference. The MPC controller outperformed the $\mathrm{PI}$ and SF in terms of settling and rising time, which allowed achieving the desired indoor $\mathrm{CO}_{2}$ level limit while satisfying the indoor comfort.

The performance of the MPC can be explained by its predictive mechanism, which includes the comfort cost and energy usage as optimization functions, for predicting the effective ventilation flow rate. It achieved $0.13 \mathrm{~m}^{3} / \mathrm{s}\left(468 \mathrm{~m}^{3} / \mathrm{h}\right)$, as depicted in Figure $3 \mathrm{~b}$. Compared to $0.135 \mathrm{~m}^{3} / \mathrm{s}$ and $486 \mathrm{~m}^{3} / \mathrm{h}$ obtained for both the PI and SF, respectively, the MPC flow rate was slightly optimized by $3.71 \%$, and this was due to the future prediction of indoor $\mathrm{CO}_{2}$ values. Regarding the power consumption of the three controllers, we computed both the instantaneous and accumulated power. Figure $3 \mathrm{c}$ shows the instantaneous power consumption required by the first standalone fan for supplying the fresh outdoor air into the controlled space. The results show that the three controllers required almost the same power, but the MPC showed a slight energy reduction.

The total power consumption for the first fan was estimated to be $598.37 \mathrm{Wh}, 611.40 \mathrm{Wh}$, and 612.76 Wh for the MPC, SF and PI, respectively, as illustrated in Figure 3d. The total power consumption demanded by the three controllers including the second fan, which exhausted the indoor air pollutant concentrations to the outside of the building, is reported in Table 3. As expected, the energy gain for the MPC strategy, when compared to both the state feedback and PI controllers, was slightly smaller.

Table 3. Energy consumption based on the MPC, PI and the state feedback control approaches.

\begin{tabular}{cccc}
\hline Energy $(\mathbf{W h})$ & PI & SF & MPC \\
\hline Total & 1225.52 & 1222.8 & 1196.74 \\
Gain & $2.35 \%$ & $2.13 \%$ & - \\
\hline
\end{tabular}

\subsection{GPC vs. MPC Performance}

This section focuses on the performance evaluation of the GPC against the MPC controller. As illustrated in Figure 4a, the GPC showed a great improvement compared to MPC with faster settling/rise times to achieve and to maintain the $\mathrm{CO}_{2}$ at the fixed setpoint. The results also showed that the designed GPC controller based on the CARIMA model provided better stability and robustness than the MPC. In fact, it was able to rapidly reach the established $\mathrm{CO}_{2}$ reference and then continue maintaining it in spite of the occupants' presence variation. This was due, in our case, to the rate weighting of the control action $\Delta u$ in the optimized criterion J. Its role was to minimize the deviation between the system's output (measured $\mathrm{CO}_{2}$ concentration) and the $\mathrm{CO}_{2}$ reference trajectory. Figure $4 \mathrm{~b}$ shows the estimated ventilation rate for both the predictive controllers according to the indoor $\mathrm{CO}_{2}$ concentration, which was generated by the occupants. As expected, the ventilation based on the GPC was able to operate firstly in order to provide the required flow rate as well as to meet the desired reference (fixed to $750 \mathrm{ppm}$ ), by anticipating the future $\mathrm{CO}_{2}$ concentration. Unlike the MPC, the ventilation flow rates provided by the GPC achieved $0.2 \mathrm{~m}^{3} / \mathrm{s}\left(720 \mathrm{~m}^{3} / \mathrm{h}\right)$ and decreased to reach $0 \mathrm{~m}^{3} / \mathrm{s}$ according to the occupants' presence. This explained the ventilation cessation behavior, which allowed a significant energy reduction during this period as illustrated in Figure 4c. In fact, the fast change in the ventilation rate behavior nicely allowed keeping the $\mathrm{CO}_{2}$ comfort constraint at the required level with little increases 
in energy consumption, compared to the MPC. However, the GPC-based CARIMA anticipates well the severity of occupancy changes and acts accordingly. As shown in Figure 4c, during unoccupied periods, the ventilators are almost at rest because of the GPC anticipation behavior.

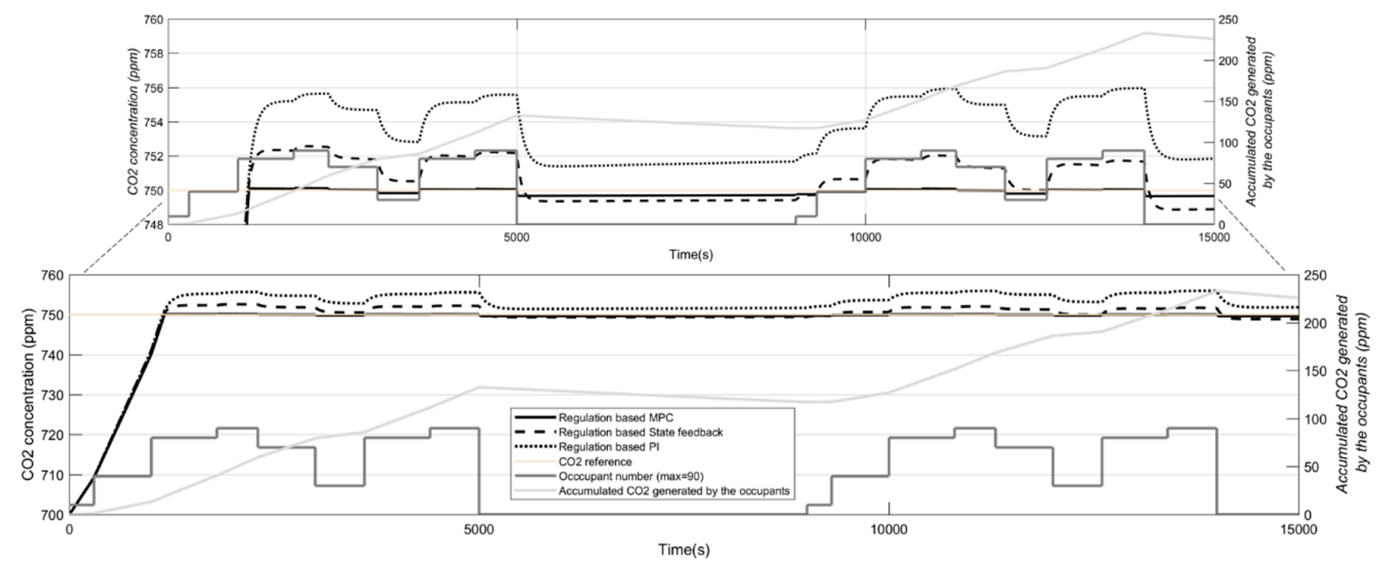

(a)

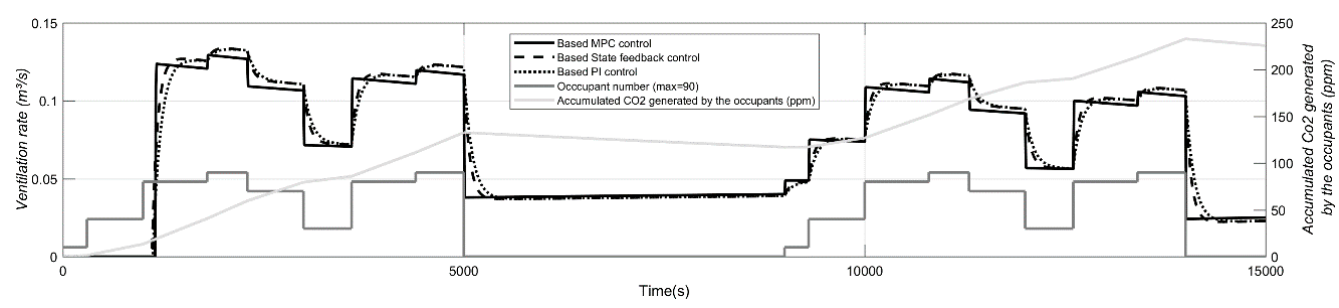

(b)

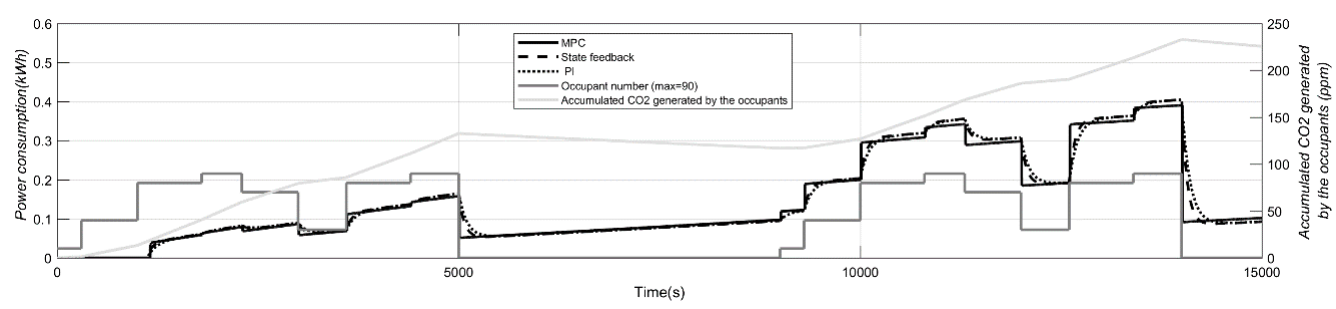

(c)

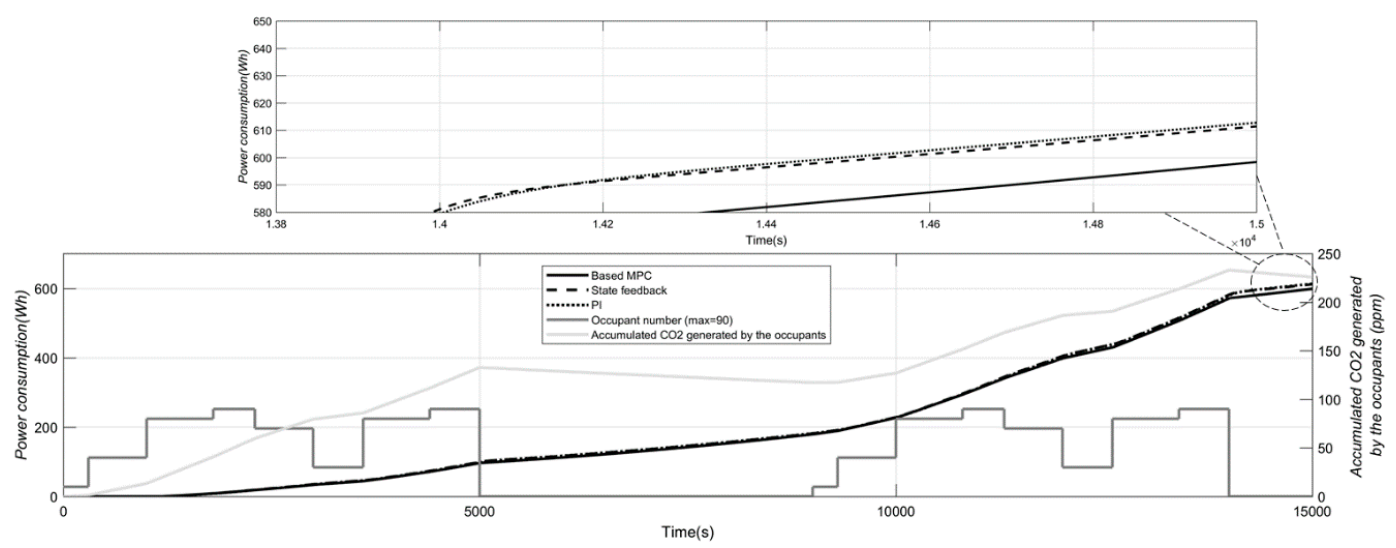

(d)

Figure 3. MPC vs. PI and state feedback (SF): (a) the CO2 regulation, (b) the ventilation air flow rate, (c) the instantaneous power consumption, and (d) the accumulated power consumption. 


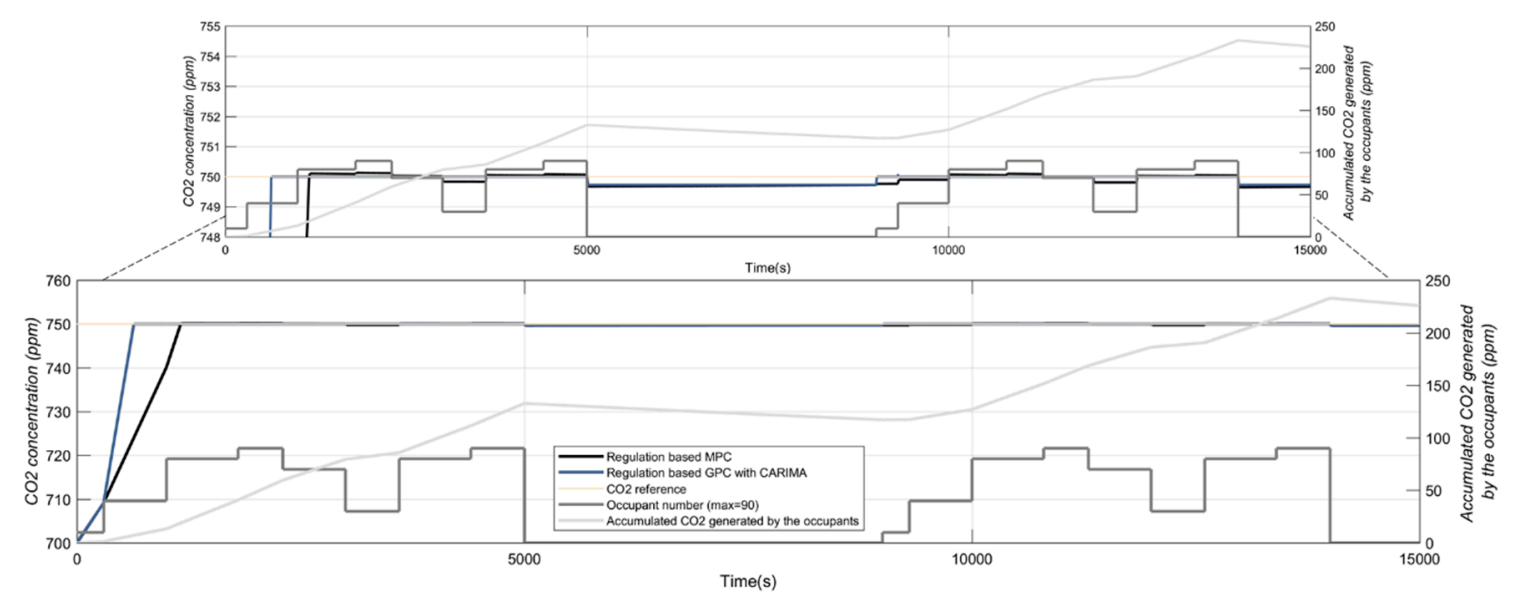

(a)

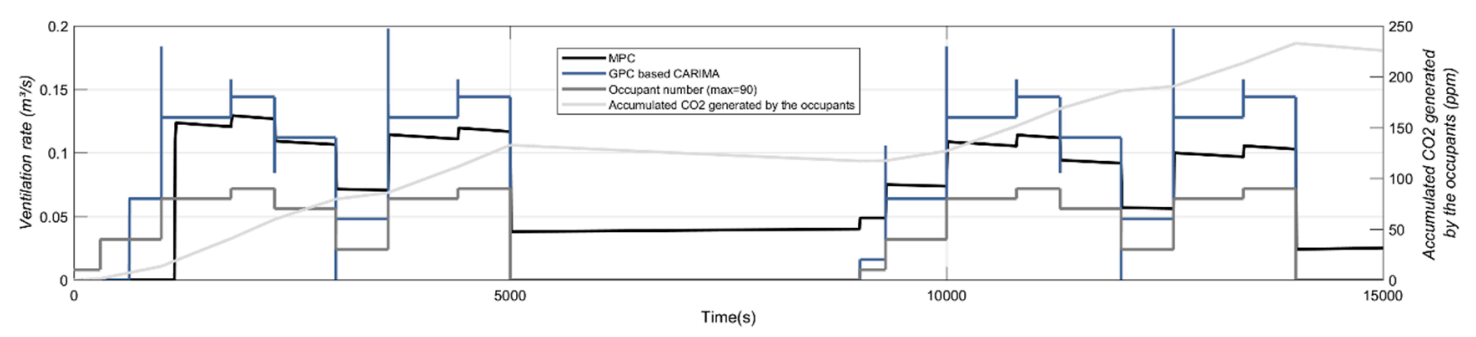

(b)

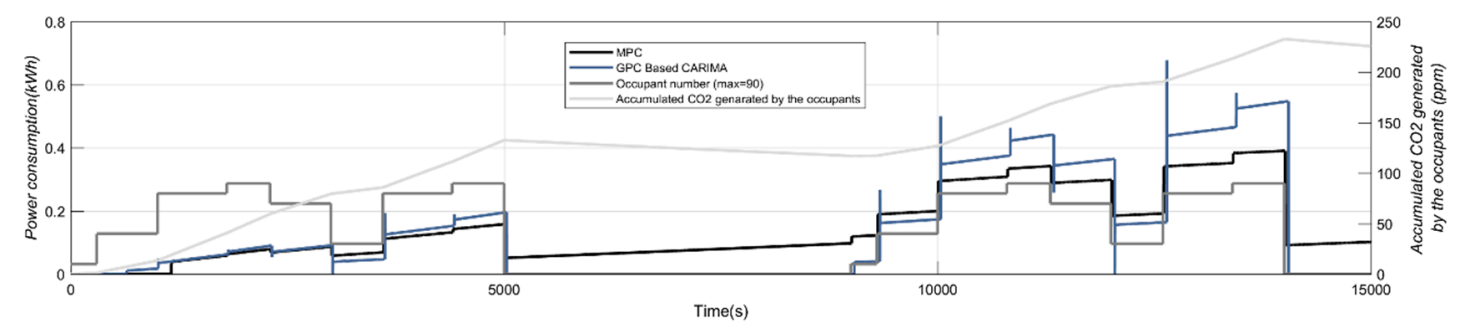

(c)

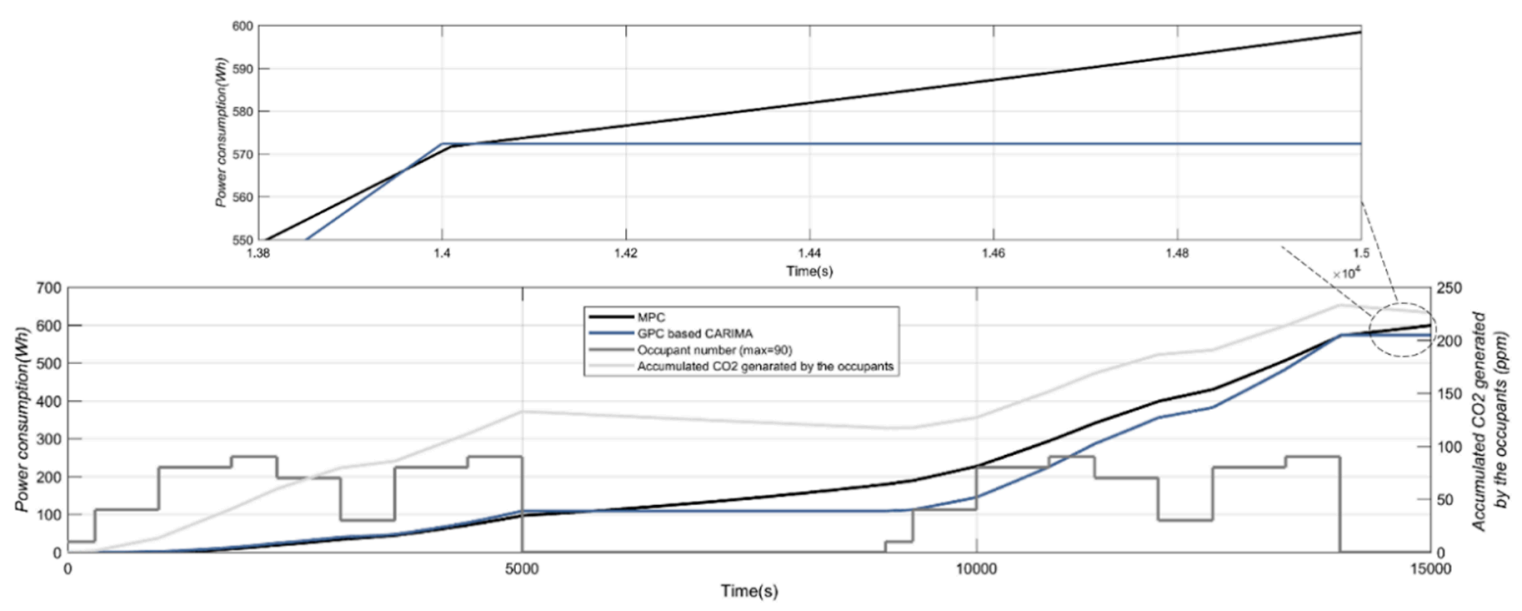

(d)

Figure 4. GPC based on CARIMA vs. the MPC: (a) the CO2 regulation, (b) the ventilation air flow rate, (c) the instantaneous power consumption, and (d) the accumulated power consumption. 
The instantaneous power consumption results for the first fan showed that the GPC allows a slight energy saving compared to the MPC, as shown in Figure 4c. Figure 4d shows that the GPC consumes about 572.39 Wh and 598.37 Wh for MPC. The total consumption (i.e., two fans) for both controllers is reported in Table 4. As expected, the energy gain for the GPC, when compared to the MPC, is slightly better.

Table 4. Total energy consumption based on the predictive controllers.

\begin{tabular}{ccc}
\hline Energy $\mathbf{( W h )}$ & MPC & GPC \\
\hline Total & 1196.74 & 1144.78 \\
Gain & $4.34 \%$ & - \\
\hline
\end{tabular}

\section{Conclusions and Perspectives}

In this work, a performance comparison between the two predictive controllers were performed and analyzed for building ventilation systems. The PI and state feedback controllers were used as baseline references to investigate the effectiveness of predictive control approaches. Both MPC and GPC control algorithms were designed and successfully implemented. The main objective was to evaluate the effectiveness of each predictive approach in terms of the indoor air quality and energy efficiency. Mainly four metrics were evaluated: the regulation of the indoor $\mathrm{CO}_{2}$ concentration, the ventilation rate, the instantaneous power consumption and the total power consumption. We conclude from the simulation results that using the GPC algorithm based on the CARIMA input/output model allowed the better performance than the MPC for improving both indoor air quality and energy conservation. In fact, compared to the MPC, the GPC provided a better stability and robustness against external disturbance introduced by the occupants. This was due to the fast mathematical derivation of its prediction parameters between the system's input/output, using the CARIMA model, and to the simplicity of integrating disturbances and constraints in the controller's structure. In terms of energy consumption, the simulation results also showed that controlling the proposed system with the GPC enabled a better reduction in the energy consumption compared to the MPC, with an overall energy gain equal to $4.34 \%$. This deviation on cumulative energy consumption was mainly related to the occupancy behavior we used together with the other parameters, especially, the prediction horizon and the set-point. This will be further investigated when deployed and experimented in real-sitting scenarios. In fact, the studied controllers will be deployed in our ventilation system, already integrated in our EEBLab. Real-time machine-learning algorithms, that we developed for occupancy forecasting, will be used to forecast $\mathrm{CO}_{2}$, which is required for the MPC and GPC in order to forecast the ventilation rates. The GPC and MPC will be also evaluated for different time horizons together with their performance for both accuracy and time processing, especially when deployed in our IoT platform's edge nodes (e.g., Raspberry PI, NVidia Nano).

Future research will focus on enhancing the controllers' order by adding the thermal comfort equation for further applications in heating, ventilation and air-conditioning system control. Both predictive control strategies will be assessed using different criteria in order to balance between the occupants' comfort (i.e., thermal and indoor air quality) and energy consumption.

Author Contributions: Conceptualization, A.B. and R.O.; methodology, R.O. and M.B.; software, A.B. and R.O.; validation, A.B., R.O. and M.B.; formal analysis, R.O.; investigation, A.B., R.O. and M.B.; resources, A.B., R.O. and M.B.; data curation, A.B. and R.O.; writing-original draft preparation, A.B.; writing-review and editing, M.E. and M.B.; visualization, A.B., R.O. and M.B.; supervision, R.O., M.E. and M.B.; project administration, M.B., M.E. and R.O. All authors have read and agreed to the published version of the manuscript.

Funding: This research was funded by «Ministere de l'Enseignement Superieur, de la Recherche Scientifique et de la Formation des Cadres (MESRSFC)» and «Centre National pour la Recherche Scientifique et Technique (CNRST)», and partially supported by MIGRID project (grant 5-398, 2017-2019), which is funded by USAID under the PEER program.

Conflicts of Interest: The authors declare no conflict of interest. 


\section{References}

1. Nejat, P.; Jomehzadeh, F.; Taheri, M.M.; Gohari, M.; Majid, M.Z.A. A global review of energy consumption, $\mathrm{CO}_{2}$ emissions and policy in the residential sector (with an overview of the top ten $\mathrm{CO}_{2}$ emitting countries). Renew. Sustain. Energy Rev. 2015, 43, 843-862. [CrossRef]

2. Del Mar-Castilla, M.; Álvarez, J.D.; Normey-Rico, J.E.; Rodríguez, F.; Berenguel, M. A multivariable nonlinear MPC control strategy for thermal comfort and indoor-air quality. In Proceedings of the IECON 39th Annual Conference of the IEEE Industrial Electronics Society, Vienna, Austria, 10-13 November 2013; pp. 7908-7913.

3. ASHRAE. Ventilation for Preservation of Occupants' Health, Safety and Wellbeing-Specify Indoor Air Quality, Minimum Ventilation Rate Acceptable for Human Occupants and will not Impair Health; 62-1990; ASHRAE: Atlanta, GA, USA, 1990.

4. European Committee for Standardization. Ventilation for Non-Residential Buildings. Performance Requirements for Ventilation and Room-Conditioning Systems; prEN-13779; CEN: Geneva, Switzerland, 2003.

5. Atthajariyakul, S.; Leephakpreeda, T. Real-time determination of optimal indoor-air condition for thermal comfort, air quality and efficient energy usage. Energy Build. 2004, 36, 720-733. [CrossRef]

6. European Committee for Standardization. EN 15251-Indoor Environmental Input Parameters for Design and Assessment of Energy Performance of Buildings Addressing Indoor Air Quality, Thermal Environment, Lighting and Acoustics; European Committee for Standardization: Geneva, Switzerland, 2007.

7. Redlich, C.A.; Sparer, J.; Cullen, M.R. Sick-building syndrome. Lancet 1997, 349, 1013-1016. [CrossRef]

8. Kharbouch, A.; Naitmalek, Y.; Elkhoukhi, H.; Bakhouya, M.; De Florio, V.; El Ouadghiri, M.D.; Latre, S.; Blondia, C. IoT and big data technologies for monitoring and processing real-time healthcare data. IJDST 2019, 10, 17-30. [CrossRef]

9. Kukadia, V.; Palmer, J. The effect of external atmospheric pollution on indoor air quality: A pilot study. Energy Build. 1998, 27, 223-230. [CrossRef]

10. Coley, D.A.; Beisteiner, A. Carbon dioxide levels and ventilation rates in schools. Int. J. Vent. 2002, 1, 45-52. [CrossRef]

11. Seppänen, O.A.; Fisk, W.J.; Mendell, M.J. Association of ventilation rates and $\mathrm{CO}_{2}$ concentrations with health andother responses in commercial and institutional buildings. Indoor Air 1999, 9, 226-252. [CrossRef]

12. El Mankibi, M. Indoor air quality control in case of scheduled or intermittent occupancy based building: Development of a scale model. Build. Environ. 2009, 44, 1356-1361. [CrossRef]

13. Chao, C.Y.; Hu, J.S. Development of an enthalpy and carbon dioxide based demand control ventilation for indoor air quality and energy saving with neural network control. Indoor Built Environ. 2004, 13, 463-475. [CrossRef]

14. Wang, S.; Sun, Z.; Sun, Y.; Zhu, N. Online optimal ventilation control of building air-conditioning systems. Indoor Built Environ. 2011, 20, 129-136. [CrossRef]

15. Lachhab, F.; Bakhouya, M.; Ouladsine, R.; Essaaidi, M. Context-driven monitoring and control of buildings ventilation systems using big data and internet of things-based technologies. Proc. Inst. Mech. Eng. Part IJ. Syst. Control Eng. 2019, 233, 276-288. [CrossRef]

16. Thosar, A.; Patra, A.; Bhattacharyya, S. Feedback linearization based control of a variable air volume air conditioning system for cooling applications. ISA Trans. 2008, 47, 339-349. [CrossRef] [PubMed]

17. Arguello-Serrano, B.; Velez-Reyes, M. Nonlinear control of a heating, ventilating, and air conditioning system with thermal load estimation. IEEE Trans. Control Syst. Technol. 1999, 7, 56-63. [CrossRef]

18. Lachhab, F.; Ouladsine, R.; Bakhouya, M.; Essaidi, M. An energy-efficient approach for controlling heating and air-conditioning systems. In Proceedings of the 2017 International Renewable and Sustainable Energy Conference (IRSEC), Tangier, Morocco, 4-7 December 2017; pp. 1-7.

19. Guo, W.; Zhou, M. Technologies toward thermal comfort-based and energy-efficient hvac systems: A review. In Proceedings of the 2009 IEEE International Conference on Systems, Man and Cybernetics, San Antonio, TX, USA, 11-14 October 2009; pp. 3883-3888.

20. Zhang, J.; Zhang, K. A particle swarm optimization approach for optimal design of PID controller for temperature control in HVAC. ICMTMA 2011, 1, 230-233.

21. Lim, D.; Rasmussen, B.P.; Swaroop, D. Selecting PID control gains for nonlinear HVAC\&R systems. HVACR Res. 2009, 15, 991-1019. 
22. Song, Y.; Wu, S.; Yan, Y.Y. Control strategies for indoor environment quality and energy efficiency-A review. Int. J. Low Carbon Technol. 2013, 10, 305-312. [CrossRef]

23. Behrooz, F.; Mariun, N.; Marhaban, M.H.; Radzi, M.; Amran, M.; Ramli, A.R. Review of control techniques for HVAC systems-Nonlinearity approaches based on Fuzzy cognitive maps. Energies 2018, 11, 495. [CrossRef]

24. Berouine, A.; Akssas, E.; Naitmalek, Y.; Lachhab, F.; Bakhouya, M.; Ouladsine, R.; Essaaidi, M. A fuzzy logic-based approach for HVAC systems control. In Proceedings of the 2019 6th International Conference on Control, Decision and Information Technologies (CoDIT), Paris, France, 23-26 April 2019; pp. 1510-1515.

25. Oldewurtel, F.; Parisiob, A.; Jones, N.C.; Gyalistrasa, D.; Gwerderd, M.; Stauche, V.; BeatLehmann, B.; Morari, M. Use of model predictive control and weather forecasts for energy efficient building climate control. Energy Build. 2012, 45, 15-27. [CrossRef]

26. Bălan, R.; Cooper, J.; Chao, K.M.; Stan, S.; Donca, R. Parameter identification and model based predictive control of temperature inside a house. Energy Build. 2011, 43, 748-758. [CrossRef]

27. Ma, J.; Qin, J.; Salsbury, T.; Xu, P. Demand reduction in building energy systems based on economic model predictive control. Chem. Eng. Sci. 2011, 67, 92-100. [CrossRef]

28. Moroşan, P.D.; Bourdais, R.; Dumur, D.; Buisson, J. Building temperature regulation using a distributed model predictive control. Energy Build. 2010, 42, 1445-1452. [CrossRef]

29. Huang, G. Model predictive control of VAV zone thermal systems concerning bi-linearity and gain nonlinearity. Control Eng. Pract. 2011, 19, 700-710. [CrossRef]

30. Xu, M.; Li, S. Practical generalized predictive control with decentralized identification approach to HVAC systems. Energy Convers. Manag. 2007, 48, 292-299. [CrossRef]

31. Privara, S.; Široký, J.; Ferkl, L.; Cigler, J. Model predictive control of a building heating system: The first experience. Energy Build. 2011, 43, 564-572. [CrossRef]

32. Yuan, S.; Perez, R. Multiple-zone ventilation and temperature control of a single-duct VAV system using model predictive strategy. Energy Build. 2006, 38, 1248-1261. [CrossRef]

33. Afram, A.; Janabi-Sharifi, F. Theory and applications of HVAC control systems-A review of model predictive control (MPC). Build. Environ. 2014, 72, 343-355. [CrossRef]

34. Bakhouya, M.; NaitMalek, Y.; Elmouatamid, A.; Lachhab, F.; Berouine, A.; Boulmrharj, S.; Ouladsine, R.; Felix, V.; Zinedine, K.; Khaidar, K.; et al. Towards a context-driven platform using IoT and big data technologies for energy efficient buildings. In Proceedings of the 2017 3rd International Conference of Cloud Computing Technologies and Applications (CloudTech), Rabat, Morocco, 24-26 October 2017; pp. 1-5.

35. Berrabah, S.; Moussa, M.O.; Bakhouya, M. Towards a thermo-mechanical characterization approach of buildings' envelope. Energy Rep. 2020, 6, 240-245. [CrossRef]

36. Elmouatamid, A.; NaitMalek, Y.; Bakhouya, M. An energy management platform for micro-grid systems using internet of things and big-data technologies. J. Syst. Control Eng. 2019, 233, 904-917. [CrossRef]

37. Boulmrharj, S.; Nait-Malek, Y.; Elmouatamid, A.; Bakhouya, M.; Ouladsine, R.; Zine-Dine, K.; Khaidar, M.; Siniti, M. Battery characterization and dimensioning approaches for micro-grid systems. Energies 2019, 12, 1305. [CrossRef]

38. Elkhoukhi, H.; Nait-Malek, Y.; Bakhouya, M.; Berouine, A.; Kharbouch, A.; Lachhab, F.; Hanifi, M.; El Ouadghiri, D.; Essaaidi, M. A platform architecture for occupancy detection using stream processing and machine learning approaches. Concurr. Comput. Pract. Exp. 2019, e5651. [CrossRef]

39. Berouine, A.; Ouladsine, R.; Bakhouya, M.; Lachhab, F.; Essaaidi, M. A model predictive approach for ventilation system control in energy efficient buildings. In Proceedings of the 2019 th World Conference on Complex Systems (WCCS), Ouarzazate, Morocco, 22-25 April 2019; pp. 1-6.

40. Allard, F.; Dorer, V.B.; Feustel, H.E. Fundamentals of the Multizone Air Flow Model-COMIS; Air Infiltration and Ventilation Centre: Coventry, UK, 1990; p. 115.

41. Zannetti, P. Air Pollution Modeling: Theories, Computational Methods and Available Software; Springer Science \& Business Media: Berlin/Heidelberg, Germany, 2013.

42. Clarke, D.W.; Mohtadi, C.; Tuffs, P.S. Generalized predictive control-Part I. The basic algorithm. Automatica 1987, 23, 137-148. [CrossRef]

43. Yang, S.H.; Wang, X.Z.; McGreavy, C. A multivariable coordinated control system based on predictive control strategy for FCC reactor-regenerator system. Chem. Eng. Sci. 1996, 51, 2977-2982. [CrossRef]

44. Qin, S.J.; Badgwell, T.A. A survey of industrial model predictive control technology. Control Eng. Pract. 2003, 11, 733-764. [CrossRef] 
45. Gunnar, B.; Folkesson, K. Recommendations for Calculations of Energy Consumption for Air Handling Units; Eurovent/Cecomaf WG 6C; Eurovent: Brussels, Belgium, 2005.

46. Benech, P.; Haessig, P. Estimation du Nombre de Personnes Presentes Dans Une Piece a Partir de la Concentration En Dioxyde De Carbone; Institute of Electronics and Telecommunications of Rennes: Rennes, France, 2015.

(C) 2020 by the authors. Licensee MDPI, Basel, Switzerland. This article is an open access article distributed under the terms and conditions of the Creative Commons Attribution (CC BY) license (http://creativecommons.org/licenses/by/4.0/). 